\title{
1 Unravelling post-transcriptional PrmC-dependent regulatory mechanisms in
}

\section{Pseudomonas aeruginosa}

3 Jonas Krueger ${ }^{1,3}$, Sarah Pohl ${ }^{1,2}$, Matthias Preusse ${ }^{1,2}$, Adrian Kordes ${ }^{1}$, Nils Rugen ${ }^{1}$, Monika

4 Schniederjans ${ }^{1,2}$, Andreas Pich $^{3 \#}$, Susanne Häussler ${ }^{1,2 \# *}$

\section{Affiliations:}

$6{ }^{1}$ Institute for Molecular Bacteriology, TWINCORE GmbH, Centre for Experimental and Clinical

7 Infection Research, a joint venture of the Hannover Medical School and the Helmholtz Centre for

8 Infection Research, Hannover, Germany.

$9{ }^{2}$ Department of Molecular Bacteriology, Helmholtz Center for Infection Research, Braunschweig, 10 Germany.

$11{ }^{3}$ Research Core Unit Proteomics and Institute of Toxicology, Hannover Medical School, Hannover, 12 Germany.

13 "Contributed equally

$14 *$ Corresponding author:

Prof Dr. med. Susanne Häussler

Institute for Molecular Bacteriology Twincore, Centre for Experimental and Clinical Infection Research, a joint venture of the Helmholtz Centre for Infection Research, Braunschweig and the Hannover Medical School Feodor-Lynen-str. 7, 30625 Hannover Tel: +49-531-6181-3000 Fax: +49-531 6181-3099

Email: susanne.haeussler@helmholtz-hzi.de

Running Title: 


\section{Originality-Significance Statement}

By applying SILAC technology in combination with mRNA-sequencing we provide evidence that the regulatory control of translation termination via the methyltransferase PrmC influences target gene expression in the opportunistic pathogen Pseudomonas aeruginosa and has a major impact on the bacterial phenotype. The finding that a bias in stop codon usage determines the $P$. aeruginosa phenotype is unexpected and adds complexity even to well-studied regulatory circuits. Modulation of PrmC activity allows the bacterial cell to cross-regulate targets independently of transcriptional signals. It represents a post-transcriptional regulatory mechanism with an underestimated but major impact on the bacterial phenotype.

\section{Summary}

Transcriptional regulation has a central role in cellular adaptation processes and is well investigated. In contrast, the importance of the post-transcriptional regulation on these processes is less well defined. The technological advancements have been critical to precisely quantify protein and mRNA level changes and hold promise to provide more insights into how post-transcriptional regulation determines phenotypes. In Pseudomonas aeruginosa the methyltransferase PrmC methylates peptide chain release factors to facilitate translation termination. Loss of PrmC activity abolishes anaerobic growth and leads to reduced production of quorum sensing-associated virulence factors. Here, by applying SILAC technology in combination with mRNA-sequencing, we provide evidence that the $P$. aeruginosa phenotype can be attributed to a change in protein to mRNA ratios of selected protein groups. The UAG-dependent translation termination was more dependent on PrmC activity than the UAA- and UGA-dependent translation termination. Additionally, we found a bias towards UAG stop codons in global transcriptional regulators. The finding that this bias in stop codon usage determines the $P$. aeruginosa phenotype is unexpected and adds complexity to regulatory circuits. Via modulation of PrmC activity the bacterial cell can cross-regulate targets independently of transcriptional signals, a process with an underestimated impact on the bacterial phenotype. 


\section{Introduction}

The ability of bacteria to adapt to various environmental conditions is one reason for their evolutionary success. A prime example for effective adaptability is the Gram-negative bacterium Pseudomonas aeruginosa, which is a ubiquitous environmental bacterium and an opportunistic human pathogen. It plays a dominant role as the causative agent of often devastating hospitalacquired infections. Furthermore, $P$. aeruginosa is frequently recovered from biofilm-associated chronic persistent infections (Bodey et al., 1983; Costerton et al., 1999; Foxman, 2010). Today, P. aeruginosa is the most prevalent pulmonary pathogen in cystic fibrosis (CF) patients (Folkesson et al., 2012). P. aeruginosa persists in the lungs of these patients within biofilms, which provide protection against antimicrobial therapy and the host immune response. Chronic respiratory tract infections in CF are associated with ongoing inflammation and changes in the structure and function of the affected organ and thus largely determine morbidity and mortality. The increasing development of multi-drug resistant $P$. aeruginosa isolates aggravates the management of acute and chronic infections and further restricts therapy options (Breidenstein et al., 2011).

P. aeruginosa is well-known for its remarkable adaptability to various and changing environmental conditions. Survival in altering habitats is largely reflected by changes in the transcriptional profile and a complex network of transcriptional regulators governs e.g. the switch of bacterial lifestyles from a unicellular planktonic to a multicellular biofilm state as well as the production of virulence factors (Jimenez et al., 2012). Although transcriptional regulation is a key step in the control of bacterial gene expression, post-transcriptional regulation seems to play a larger role than previously expected. Examples of post-transcriptional regulators are small regulatory RNAs (sRNAs) or the CsrA/RsmA system which both are involved in highly sophisticated regulatory circuits (Wassarman, 2002; Ventre et al., 2006; Brencic and Lory, 2009; Romeo et al., 2013). Also the modification of the translation machinery can affect RNA processing and thus represents a post-transcriptional regulatory mechanism (Pustelny et al., 2013; Shi et al., 2015; Little et al., 2016). The methyltransferase PrmC methylates class one peptide chain release factors (PrfA, PrfB) on a 
glutamine residue of the GGQ motif in various bacteria including Escherichia coli and $P$. aeruginosa (Heurgue-Hamard et al., 2002; Nakahigashi et al., 2002; Scarlett et al., 2003; Pustelny et al., 2013). These release factors are responsible for the termination of translation at the stop codons UGA, UAA and UAG (Mora et al., 2007). Deletion of PrmC results in inefficient translation termination and thus downstream sequences are translated which may also include frameshifts (Nakahigashi et al., 2002; Mora et al., 2007). However, rather than generally determining bacterial fitness and growth as a result of inefficient translation termination, loss of $\mathrm{PrmC}$ seems to affect specifically bacterial pathogenicity and environmental adaptability. For example a VagH (PrmC-orthologue) deficient Yersinia pseudotuberculosis mutant was demonstrated to exhibit an avirulent phenotype similar to a type 3 secretion system (T3SS) negative mutant, and thus it was suggested that absence of VagH affects expression of the T3SS (Garbom et al., 2007). In addition, prmC gene expression was shown to be increased in Porphyromonas gingivalis and E. coli under in vivo conditions (Park et al., 2004; John et al., 2005), indicating a potential regulatory function of $\operatorname{PrmC}$ that drives pathogenicity. In $P$. aeruginosa, PrmC deficiency resulted in a quorum sensing (QS)-independent reduction of virulence factor synthesis, e.g. pyocyanine, rhamnolipids and a lower production of secreted toxins (Pustelny et al., 2013). However, how PrmC selectively affects pathogenicity in $P$. aeruginosa remained unknown.

In order to address this question, we used stable isotope labeling by amino acids in cell culture (SILAC) in combination with shotgun proteomics and data dependent mass spectrometry (MS) analysis to determine the impact of PrmC on global $P$. aeruginosa protein biosynthesis. SILAC is a metabolic labeling strategy and well-established for quantitative proteome analysis in eukaryotic cells (Ong et al., 2002), whereas its application for the analysis of bacterial systems has remained very limited (Soufi et al., 2010; Ping et al., 2013; Jung et al., 2014; Boysen et al., 2015; Chua et al., 2016). In this study we adapted the SILAC technique to quantify even small magnitude effects in the P. aeruginosa proteome and characterized global effects of PrmC deficiency. This comparative proteome analysis was furthermore combined with RNA-seq data in order to precisely decipher 
transcriptional and post-transcriptional PrmC-dependent changes and thus provide a detailed view on the mode of action of PrmC.

\section{Results}

Previous studies revealed a strikingly attenuated phenotype of the $\operatorname{PrmC}$ deficient $P$. aeruginosa PA14 mutant in the Galleria mellonella infection model (Pustelny et al., 2013). This might be explained by a reduced production of many QS-dependent virulence factors despite normal levels of the QS signaling molecules and/or the inability of the mutant to grow under anaerobic conditions (Pustelny et al., 2013). In order to gain more insights into the molecular mechanisms underlying the attenuation of the PrmC deficient $P$. aeruginosa PA14 mutant, we adapted the metabolic labeling strategy SILAC in combination with shotgun proteomics in the $P$. aeruginosa PA14 strain.

\section{Adaption of SILAC based protein quantification in $P$. aeruginosa}

Sufficient and efficient labeling of peptides by stable isotopic labeled amino acids is an absolute prerequisite for global protein quantification using SILAC. Auxotrophy for the amino acids lysine and arginine ensures high incorporation rates of exogenously added stable isotopic labeled equivalents in eukaryotes (Ong et al., 2002; Dreisbach et al., 2008). Therefore, the first $P$. aeruginosa SILAC experiments in this study were performed in a PA14 $\Delta / y s A \Delta \arg B$ double mutant, which is auxotroph

for both amino acids. However, when ${ }^{13} \mathrm{C}_{6}{ }^{15} \mathrm{~N}_{2}$-L-lysine (lys8) and ${ }^{13} \mathrm{C}_{6}{ }^{15} \mathrm{~N}_{4}$-L-arginine (arg10) were exogenously added, the identification rate of proteins decreased in this auxotrophic mutant over time (Figure S1). Since a successful application of lys8 protein labeling has previously been described for prototroph yeast and E. coli (Frohlich et al., 2013), we next fed the PA14 wild-type strain with lys8 only. However, again no stable identification rates could be obtained (Figure S1). To avoid lysine degradation and reincorporation of heavy stable isotopes into amino acids other than lysine, the PA14 tnldcC transposon mutant from the Harvard PA14 mutant library was selected (Liberati et al., 2006), which lacks a functional LdcC protein involved in degradation of lysine (Chou et al., 2010). The number of identified proteins remained constant following lys8 protein labeling in this mutant and 
the stability of stable isotopic labeled lysine was ensured (Figure S1). By extending the exposure time of the bacteria to the labeled amino acid a SILAC incorporation efficiency of $98 \%$ was reached that nearly reflected the isotopic purity of ${ }^{15} \mathrm{~N}$ and ${ }^{13} \mathrm{C}$ in lys8. In conclusion, lys 8 was used for protein labeling and combined with LysC digestion (Figure S2) for quantitative proteome analysis in $P$. aeruginosa.

\section{Robustness of SILAC to quantify even small magnitude effects in the $P$. aeruginosa}

\section{proteome}

We next investigated the robustness of SILAC based protein quantification in $P$. aeruginosa and determined the overall degree of variation of the quantified proteins between biological replicates. We therefore grew two PA14 tnldcC cultures under identical conditions and supplemented one with unlabeled (lys 0 ) and the other with labeled (lys 8 ) lysine. We extracted the proteins from lys0 and lys 8 labeled bacteria, mixed them in a 1:1 ratio and subjected them to shotgun proteomics to determine labeled/unlabeled protein ratios. We calculated the threshold for reliable fold-changes in protein abundance to be a ratio of 1.2 which corresponds to a $95 \%$ confidence interval of all protein ratios (Figure S3).

\section{Quantitative, PrmC dependent proteome analysis}

We used the adapted SILAC technique to evaluate whether production of $P$. aeruginosa proteins and particular those that drive pathogenicity were affected by expression of the release factor methyltransferase PrmC. We therefore performed comparative proteomics of the PA14 tnprmC strain harboring the empty vector as control (tnprmC) against the PA14 tnprmC mutant complemented with the prmC gene in trans (tnprmC::prmC) in order to ensure measurement of PrmC-dependent effects in the identical strain background. The lys 8 labeled proteins extracted from the PA14 tn/dcC mutant (tn/dcC) were used as "spike in" standard (Geiger et al., 2011). Therefore the tnprmC and tnprmC::prmC samples were mixed with the heavy labeled reference and analyzed individually by liquid chromatography (LC)-MS/MS analysis. Ratios between the heavy labeled "spike 

in" proteins and the unlabeled proteins from the samples were generated and compared to determine the final ratios for the relative protein abundance of the tnprmC versus tnprmC::prmC samples (Fig. 1). Replicates were plotted against each other to evaluate the biological reproducibility of protein changes. All replicates showed high correlation (Squared Pearson's correlation coefficient of 0.87-0.93, Figure S4).

Among the overall 2722 detected and quantified proteins, 1699 proteins were found in all three replicates of the tnprmC and tnprmC::prmC strains (listed in Table S1). Contrary to the 2D-based proteome analysis, which revealed only 26 quantified proteins (Pustelny et al., 2013), this extensive data set enables global similarity analysis of termination sequences. In the SILAC based analysis we observed 94 proteins with a significant higher abundance and 296 proteins with a significant lower abundance in the tnprmC mutant ( $p$-value $\leq 0.05$; Fig. 2 ). This highlights that in general PrmC deletion negatively affects the protein translation in $P$. aeruginosa. In accordance with the previous observation of a lower virulence factor production in tnprmC (Pustelny et al., 2013), the mutant produced lower levels of e.g. proteins involved in phenazine biosynthesis (PhzM, PhzB1, PhzB2, PhzS, PhzE2, PhzD2; Table S1) and of enzymes for rhamnolipid synthesis (RhlB, RmIC; Table S1).

\section{Proteome-transcriptome correlation}

Changes on protein level in the tnprmC mutant may be the direct result of a translational termination deficiency. However, if protein levels of e.g. transcriptional regulators were affected, those might transcriptionally affect downstream targets and thus may lead to lower corresponding protein levels as a secondary effect of inefficient translation termination. We therefore also acquired RNA-seq data in order to examine the correlation between protein and mRNA abundance (Table S2).

We first compared the mRNA data of the tnprmC::prmC strain with its global protein quantification data (Table S3). A correlation analysis revealed a squared Pearson's correlation coefficient of 0.20 of protein to mRNA abundance (Figure S5). Nevertheless, in the group of genes that exhibited a high protein to mRNA ratio (upper $25 \%$ of the genes) the functional PseudoCAP categories "amino acid 
synthesis and metabolism", "energy metabolism" and "fatty acid and phospholipid metabolism" were enriched. Vice versa in the group of genes that exhibited a low protein to mRNA ratio (lower $25 \%$ of the genes) the functional categories "secreted factors (toxins, enzymes, alginate)", "transcriptional regulators" and "two component regulatory system" were enriched (Figure S5).

We also recorded the transcriptional profile of tnprmC mutant and compared it to the RNA-seq data of tnprmC::prmC strain. We observed 90 genes with a significantly higher expression and 183 genes with a significantly lower expression in the tnprm C mutant (adjusted p-value $\leq 0.05$, (Anders and Huber, 2010), genes are listed in Table S2). Among the latter were genes involved in phenazine biosynthesis and other QS-regulated genes. This indicates that the lower levels of QS-dependent virulence factors in the prmC mutant are due to lower mRNA abundances and not necessarily due to an inefficient translation termination of the respective mRNAs.

In order to identify those proteins that are directly post-transcriptionally influenced by PrmC, the protein to mRNA correlation ratios were calculated for tnprmC and tnprmC::prmC. Therefore, all quantified proteins (1699) were assigned to the corresponding mRNA data. The high correlation coefficient $\left(r^{2}=0.92\right)$ indicates that most of the ratios were independent of the presence of PrmC. However, some proteins diverged and exhibited PrmC-dependent higher or lower protein abundance in relation to the level of the corresponding mRNA (Fig. 3).

One would expect a lower protein to mRNA ratio if mRNA was not sufficiently translated due to inefficient translation termination in the tnprmC mutant. Those 100 genes that exhibited the lowest PrmC-dependent ratio between protein and mRNA (top100 low) are listed in Table S4. The 100 genes with the highest ratio between protein and mRNA are listed in Table S5 (top100 high). Interestingly, genes of the functional PseudoCAP category "Chaperones \& heat shock proteins" were highly enriched in the top100 high group, whereas genes of the functional category "Transport of small molecules" were enriched in the top100 low group (Figure S6). 
Among the top100 low group of proteins we did not identify a transcriptional regulator of one of the

P. aeruginosa QS-systems that might directly explain the lower production of virulence factors in the tnprmC mutant. However, the global regulator DNR was identified at lower protein levels in the tnprmC mutant. DNR controls the expression of several denitrification genes (Giardina et al., 2011). Under anaerobic conditions, nitrate or nitrite are used as the terminal electron acceptor in $P$. aeruginosa. Since the tnprmC mutant has previously been shown to be severely affected under anaerobic conditions, we complemented the tnprmC mutant with the DNR gene in trans (tnprmC::dnr) and determined growth under anaerobic conditions. As shown in Fig. $4 A$ the tnprm $C$ mutant could be indeed partially rescued by $d n r$ complementation under anaerobic growth conditions in glucose containing medium. Incomplete complementation might be explained by inefficient $d n r$ translation even if the transcript level is increased. However, restoration of anaerobic growth via $d n r$ expression in trans was not sufficient to compensate the lower virulence of the tnprmC mutant in the Galleria mellonella infection model (Fig. 4B). Interestingly the infection assay revealed that pyocyanine production is not required for full pathogenicity.

\section{prmC is differentially regulated under diverse environmental conditions}

217 If the activity of PrmC impacts on post-transcriptional regulatory processes, it is reasonable to assume that $p r m C$ itself might be subject to regulation by environmental cues. We therefore reanalyzed previously recorded data on mRNA expression levels in PA14 grown under 14 different conditions (Dotsch et al., 2015). Indeed, prmC was significantly differentially expressed under following conditions as compared to exponential growth phase (in LB medium): stationary growth phase, attachment experiments in M9 minimal medium (Godeke et al., 2012), heat shock at $42{ }^{\circ} \mathrm{C}$ or $50{ }^{\circ} \mathrm{C}$, in vivo growth within a mouse tumor and iron deficiency (Figure S7). The changes in prmC transcript level could be caused by a differential in transcription initiation or in mRNA stability. We also quantified prmC mRNA levels in 369 clinical $P$. aeruginosa isolates. There was a high coefficient of variation (CV) for prmC of 0.36 across all isolates. This CV value was significantly higher than that of six housekeeping genes ( $p r o C, r p o D, n u o D, p p s A$, nadB, mutL) (Figure S8). Remarkably, heat shock 
proteins were enriched in the top100 high group of PrmC-dependent protein to mRNA ratios. Thus, as $\mathrm{prmC}$ expression is affected by heat shock, this regulatory mechanism might allow for an optimized translation efficiency of heat shock proteins after heat shock treatment.

\section{The UAG stop codon is enriched in genes with low PrmC-dependent protein to mRNA ratio}

Since PrmC is involved in the activation of peptide chain release factors that are important to terminate translation at the UAG, UAA, or UGA stop codons, we explored whether there is a difference in the frequency of the three stop codons in the top100 low versus the top100 high group of genes. Indeed, the UAG codon was enriched in the top100 low group. Thus, overall efficient UAGdependent translational termination of mRNAs seemed to be more dependent on the methylation of peptide chain release factors than UAA- and UGA-dependent translation termination (Fig. 5). Next, distinct functional PseudoCAP gene categories were analyzed for their stop codon preference. Interestingly, an enrichment of the UAG stop codon was found in the category "transcriptional regulators" (Figure S9). This enrichment is particularly noteworthy, since changes in the abundance of transcription factors have major effects on the cellular protein profile. Concordant with the PseudoCAP enrichment of the category "chaperones \& heat shock proteins" in the top100 high group, the analysis revealed a low UAG but a high UAA stop codon frequency, indicating that the UAA stop codon is less associated with PrmC deficiency.

\section{PrmC deletion results in higher readthrough rates of translation associated proteins}

The MS-raw data were reanalyzed and scanned for C-terminal successional peptides to determine whether global readthrough events were more frequent in PA14 tnprmC. In total, 273 proteins with readthrough events were detected; 63 readthrough peptides were found in frame, 97 with a +1 frameshift and 153 with a +2 frameshift. All these peptides were identified as specific Lys $C$ peptides and had an average Andromeda peptide ion score of 109 indicating highly specific detection (Cox et al., 2011). The intensities of readthrough peptides of the tnprmC strain were compared with that of tnprmC::prmC to identify whether they are PrmC-dependent. Despite the role of PrmC in the 
activation of the peptide chain release factor, most of these readthrough events were detected independently of prmC expression. Nevertheless, 23 readthrough events could be detected exclusively in the PA14 tnprmC strain in at least two of three replicates (in frame: 5 proteins; +1 frameshift: 9 proteins; +2 frameshift: 9 proteins; Table S6, Table S7, Table S8), whereas only three readthrough events were detected exclusively in at least two replicates of the tnprmC::prmC strain (in frame: 0, +1 frameshift: 2 and +2 frameshift: 1). Of note, 18 of these PrmC deficiency dependent peptides, were detected in the low molecular weight fraction of the SDS gel and thus were separated from the mature proteins. This indicates that proteins with readthrough events upon PrmC deletion are more likely degraded by endogenous proteases.

\section{Discussion}

The capability of $P$. aeruginosa to strive in various and challenging habitats is facilitated by its versatile metabolic capacity, which is controlled by environment-driven flexible changes in the transcriptional profile. Although transcriptional regulation is the major key in control of bacterial gene expression, post-transcriptional regulation seems to play a much larger role than previously anticipated.

With the aim to shed more light on post-transcriptional regulation in the opportunistic human pathogen $P$. aeruginosa, we adapted SILAC technology to precisely quantify protein abundances in this organism and analyzed the effect of PrmC on protein and corresponding mRNA levels. PrmC methylates class one peptide chain release factors (PrfA, PrfB) which control efficient termination of translation (Heurgue-Hamard et al., 2002; Nakahigashi et al., 2002; Pustelny et al., 2013). Altered PrmC protein levels are therefore expected to result in global proteomic changes. We have previously characterized the $P$. aeruginosa PA14 tnprmC mutant (Pustelny et al., 2013) which did not exhibit a growth defect under aerobic conditions. However, the PrmC protein was found to be essential for growth under anaerobic conditions and pathogenicity of $P$. aeruginosa in the Galleria mellonella infection model (Pustelny et al., 2013). 
In this study, we detected and quantified as many as 1910 proteins in $P$. aeruginosa PA14 following

279 lys8 labeling in a LC-MS/MS based shotgun proteome analysis. Remarkably, auxotrophy for lysine was not necessary to reach incorporation rates of more than $98 \%$. These results are in accordance with a study of Fröhlich et al. demonstrating that stable isotopic labeled lysine can be used as a labeling strategy for protein quantification experiments of prototroph microorganisms (Frohlich et al., 2013). However, in this study the prevention of $P$. aeruginosa lysine degradation seemed to be important to ensure stable labeling with lys8.

Various studies in bacteria and eukaryotes revealed a rather low correlation of protein and mRNA levels, which might indicate the importance of post-transcriptional regulation (Maier et al., 2009; Wu et al., 2013; Kwon et al., 2014; Borirak et al., 2015). In this study, the application of RNA-seq and SILAC-based protein quantification allowed for the detailed analysis of mRNA to protein ratios in $P$. aeruginosa and uncovered direct and indirect PrmC induced changes in the protein profile. The overall high correlation of the ratio of mRNA to protein levels in the prmC mutant as compared to the complemented $P$. aeruginosa strain clearly demonstrated that PrmC-dependent changes on translation efficiencies of mRNAs is not a common effect on all proteins but is rather selective. Our study additionally revealed that $p r m C$ is differentially regulated under various environmental conditions, e.g. after heat shock treatment. Remarkably, heat shock proteins were enriched in the top100 high group of PrmC-dependent protein to mRNA ratios. Thus, this regulatory mechanism seems to allow for an optimized translation efficiency of heat shock proteins after heat shock treatment.

Global regulators have recently been suggested to be subject of extensive post-transcriptional regulation in bacteria (Schmidt et al., 2016). Here we found that translation of the mRNA of selected genes was dependent on PrmC function. This bias can at least in part be explained by a differential use of stop codons in affected mRNA. Intriguingly, on a global scale the use of stop codon UAG entailed a lower and the UAA stop codon a trend to a higher PrmC-dependent protein to mRNA ratio. 
finding that the UAG stop codon is responsible for a differential translational termination efficiency upon PrmC deletion is supported by studies of Mora et al. (Mora et al., 2007). They clearly presented that the readthrough rate in E. coli strongly increases upon PrmC deletion if UAG terminates the open reading frame. Despite the general trend, not all of the mRNAs encoding for proteins that were negatively affected by PrmC deficiency harbored UAG as the stop codon and not all mRNAs that were more efficiently translated harbored the UAA stop codon. Other factors play a role in the PrmCdependent changes on protein to mRNA ratios. The identity of the adjacent codons might very well aggravate or mitigate the effect of PrmC-dependent translation termination efficiency at the various stop codons. Furthermore, C-terminal elongation of proteins due to stop codon readthrough might be neutral, but they might also stabilize or destabilize the protein. Moreover, the readthrough might not be complete and, for an individual protein, a fraction might be correctly terminated (Mora et al., 2007). This could vary from protein to protein and also under changing environmental conditions. In P. aeruginosa at least some of the PrmC-dependent changes in protein abundance seem to be due to a destabilization of the protein, since we detected the major part of readthrough peptides upon PrmC deletion in the lower molecular weight fraction. The artificially extended proteins may also be nonfunctional because of misfolding. Notably, Beznoskov et al. and Eswarappa et al. reported programmed readthrough in yeast and mammalian endothelial cells, respectively (Eswarappa et al., 2014; Beznoskova et al., 2015). Their results indicate that certain proteins acquire new or differing functions by C-terminal extension. This could also apply for bacterial proteins and would indicate new ways of post-transcriptional regulatory mechanisms.

In conclusion, by using advanced protein profiling technology we uncovered a mechanism of gene regulation that is based on a bias in stop codon usage in selected functional groups of genes. Modulating the activity of PrmC and thus readthrough events at defined stop codons that impact protein stability adds complexity even to well-studied regulatory circuits. This strategy allows the bacterial cell to cross-regulate targets independently of transcriptional signals, a process with an underappreciated but major impact on the bacterial phenotype. 


\section{Bacterial strains, media and growth conditions}

332

Strains, plasmids, and primers that were used in this study are listed in Table S9. All chemicals were provided by Sigma-Aldrich or Carl Roth unless otherwise stated. PA14 strains were cultured in modified M9 medium containing $42.2 \mathrm{mM} \mathrm{Na}_{2} \mathrm{HPO}_{4} \times 2 \mathrm{H}_{2} \mathrm{O}, 22 \mathrm{mM} \mathrm{KH}_{2} \mathrm{PO}_{4}, 18.7 \mathrm{mM} \mathrm{NH}{ }_{4} \mathrm{Cl}, 8.6 \mathrm{mM}$ $\mathrm{NaCl}, 1 \mathrm{mM} \mathrm{MgSO}_{4}, 0.1 \mathrm{mM} \mathrm{CaCl}_{2}, 20 \mathrm{mM}$ Glucose, $10 \mu \mathrm{M}$ Fe(II)sulfate and $0.1 \mathrm{mM}$ of each of the 20 canonical amino acids except lysine and arginine unless otherwise stated. Isotopic labeled amino acids or unlabeled counterparts were used at the following concentrations: ${ }^{13} \mathrm{C}_{6}{ }^{15} \mathrm{~N}_{2}$-L-lysine (lys8): $1.368 \mathrm{mM} ;{ }^{13} \mathrm{C}_{6}{ }^{15} \mathrm{~N}_{4}$-L-arginine (arg10, both SILANTES): $0.949 \mathrm{mM}$. For anaerobic growth experiments, strains were precultivated in Luria broth (LB) medium. Washed and concentrated precultures were used to inoculate main cultures in Hungate tubes containing PYG- $\mathrm{KNO}_{3}$ medium (modified from http://www.dsmz.de/microorganisms/medium/pdf/DSMZ_Medium104.pdf: $\quad 20$ g/l $\quad$ casein hydrolysate, $10 \mathrm{~g} / \mathrm{l}$ yeast extract, $10 \mathrm{~g} / \mathrm{l}$ glucose, $500 \mathrm{mg} / \mathrm{l}$ cysteine- $\mathrm{HCl}, 10 \mathrm{mg} / \mathrm{l} \mathrm{CaCl}{ }_{2} \times 2 \mathrm{H}_{2} \mathrm{O}$,

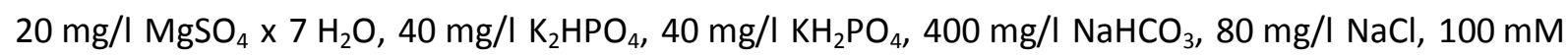
$\mathrm{KNO}_{3}$, distributed under $\mathrm{N}_{2}$ ) to a start $\mathrm{OD}_{600}$ of 0.01 . Growth was monitored by taking samples of $100 \mu \mathrm{l}$ with a cannula and subsequent $\mathrm{OD}_{600}$ measurement. Incorporation efficiency of lys 8 into the proteome of the $\mathrm{tn} / \mathrm{dcC}$ mutant was determined by preparation of $10 \mathrm{~mL}$ precultures in modified $\mathrm{M} 9$ medium either supplemented by lys 0 or lys 8 . Each preculture was used to inoculate a main culture supplemented with lys8 with a start $\mathrm{OD}_{600}$ of 0.05 . Cells were harvested after 6 and 9.5 hours incubation at $37^{\circ} \mathrm{C}$ and $180 \mathrm{rpm}$. Each sample was prepared in triplicates. To obtain protein extracts and mRNA samples for comparative quantitative LC-MS/MS analysis and RNA-seq, cultures of the strains tnprmC and tnprmC::prmC were harvested at an $\mathrm{OD}_{600}$ of 2 . To generate heavy labeled protein as internal standard, tn/dcC cells were grown in the same defined M9 medium but containing stable isotope labeled lys 8 instead of lys 0 up to an $\mathrm{OD}_{600}$ of 2 . Before protein extraction, the tnprmC and the complemented mutant cells were mixed 1:1 with lys8 labeled tn/dcC "spike-in". Carbenicillin was added to a final concentration of $400 \mu \mathrm{g} / \mathrm{ml}$ to strains harboring the pUCP20 plasmid. 

peptide samples was performed as described previously (Schroder et al., 2015) with minor changes using a reversed phase nanoflow ultrahigh pressure liquid chromatography (RSLC) system (Thermo Fisher Scientific) and an LTQ Orbitrap Velos mass spectrometer (Thermo Fisher Scientific). The raw files generated by LC-MS/MS analysis were further processed using the MaxQuant software package (version 1.5.2.8) (Cox and Mann, 2008). Mapping of detected fragments to peptide sequences was performed by the implemented search engine Andromeda (Cox et al., 2011) using a self-made database of combined $P$. aeruginosa strain PA14 annotated entries of UniProt database and Pseudomonas Genome Database (Winsor et al., 2011; UniProt, 2014). The resulting txt-file containing the identified proteins was further processed by spreadsheet application Excel (Microsoft). For more detailed information see Materials and Methods S1.

\section{Comparative transcriptome analysis}

RNA samples were extracted from the same $P$. aeruginosa cultures as the samples for proteome analysis. RNA was extracted from cell pellets using the RNeasy Mini Kit (Qiagen) in combination with Qiashredder columns (Qiagen) according to the manufacturer's instruction with some modifications. For removal of ribosomal RNA the Ribo-Zero Bacteria Kit (Illumina) was used and cDNA libraries were generated with the ScriptSeq v2 Kit (Illumina). The samples were sequenced in single end mode on an Illumina HiSeq 2500 device involving 50 cycles. Mapping was performed using stampy (Lunter and

377 Goodson, 2011) and testing for differential expression was performed with the R package DESeq 378 (Anders and Huber, 2010). For transcriptome analysis only two biological replicates for each strain were used. Adjusted p-values for differential gene expression were calculated as described by Anders and Huber (Anders and Huber, 2010). 
382

383

384

385

386

387

388

389

390

391

392

393

394

395

396

397

398

399

400

401

402

403

In order to identify readthrough events upon prmC deletion, raw files of the proteome analysis were reanalyzed by MaxQuant. Therefore, multiplicity was set to 1 . Instead of the existing PA14 database containing known coding sequences, three new databases were prepared containing the in frame, +1 frameshift and +2 frameshift protein sequences starting from the amino acid after the first lysine downstream of the stop codon to at least 50 amino acids downstream of the stop codon plus the sequence to the next lysine of the translated non-coding region. Only peptide sequences without stop codons and only unlabeled peptides were considered. For comparison of tnprmC and tnprmC::prmC, only proteins with detected peptides with intensities in at least two of the three tnprmC replicates and no intensities in all of the tnprmC::prmC replicates were considered.

\section{Acknowledgement}

We thank Karsten Heidrich for technical support, Stephan Brouwer and Christian Pustelny for providing the PA14 tnprmC and tnprmC::prmC strains and Piotr Bielecki for providing the PA14 $\triangle$ lysA $\triangle \arg B$ double mutant. We also thank A. Bielecka for library preparation for RNA sequencing. This work was supported by an European Research Council starter grant (http://erc.europa.eu/, 260276), the German Research Foundation (https://www.mhhannover.de/sfb900.html, DFG SFB 900) and Networking Fund of the Helmholtz Association of German Research Centers (HGF) under contract number VH-GS-202. The funders had no role in study design, data collection and analysis, decision to publish, or preparation of the manuscript. All authors have declared that no competing interests exist.

\section{References}

Anders, S., and Huber, W. (2010) Differential expression analysis for sequence count data. Genome Biol 11: R106. 

$427 \quad 1372$.

428 Cox, J., Neuhauser, N., Michalski, A., Scheltema, R.A., Olsen, J.V., and Mann, M. (2011) Andromeda: a 429

Beznoskova, P., Wagner, S., Jansen, M.E., von der Haar, T., and Valasek, L.S. (2015) Translation initiation factor elF3 promotes programmed stop codon readthrough. Nucleic Acids Res 43: 50995111.

Bodey, G.P., Bolivar, R., Fainstein, V., and Jadeja, L. (1983) Infections caused by Pseudomonas aeruginosa. Rev Infect Dis 5: 279-313.

Borirak, O., de Koning, L.J., van der Woude, A.D., Hoefsloot, H.C., Dekker, H.L., Roseboom, W. et al. (2015) Quantitative proteomics analysis of an ethanol- and a lactate-producing mutant strain of Synechocystis sp. PCC6803. Biotechnol Biofuels 8: 111.

Boysen, A., Borch, J., Krogh, T.J., Hjerno, K., and Moller-Jensen, J. (2015) SILAC-based comparative analysis of pathogenic Escherichia coli secretomes. J Microbiol Methods 116: 66-79.

Breidenstein, E.B., de la Fuente-Nunez, C., and Hancock, R.E. (2011) Pseudomonas aeruginosa: all roads lead to resistance. Trends Microbiol 19: 419-426.

Brencic, A., and Lory, S. (2009) Determination of the regulon and identification of novel mRNA targets of Pseudomonas aeruginosa RsmA. Mol Microbiol 72: 612-632.

Chou, H.T., Hegazy, M., and Lu, C.D. (2010) L-lysine catabolism is controlled by L-arginine and ArgR in Pseudomonas aeruginosa PAO1. J Bacteriol 192: 5874-5880.

Chua, S.L., Yam, J.K., Hao, P., Adav, S.S., Salido, M.M., Liu, Y. et al. (2016) Selective labelling and eradication of antibiotic-tolerant bacterial populations in Pseudomonas aeruginosa biofilms. Nat Commun 7: 10750.

Costerton, J.W., Stewart, P.S., and Greenberg, E.P. (1999) Bacterial Biofilms: A Common Cause of Persistent Infections. Science 284: 1318-1322.

Cox, J., and Mann, M. (2008) MaxQuant enables high peptide identification rates, individualized p.p.b.-range mass accuracies and proteome-wide protein quantification. Nat Biotechnol 26: 1367peptide search engine integrated into the MaxQuant environment. J Proteome Res 10: 1794-1805. 
Dotsch, A., Schniederjans, M., Khaledi, A., Hornischer, K., Schulz, S., Bielecka, A. et al. (2015) The Pseudomonas aeruginosa Transcriptional Landscape Is Shaped by Environmental Heterogeneity and Genetic Variation. MBio 6: e00749.

Dreisbach, A., Otto, A., Becher, D., Hammer, E., Teumer, A., Gouw, J.W. et al. (2008) Monitoring of changes in the membrane proteome during stationary phase adaptation of Bacillus subtilis using in vivo labeling techniques. Proteomics 8: 2062-2076.

Eswarappa, S.M., Potdar, A.A., Koch, W.J., Fan, Y., Vasu, K., Lindner, D. et al. (2014) Programmed translational readthrough generates antiangiogenic VEGF-Ax. Cell 157: 1605-1618.

Folkesson, A., Jelsbak, L., Yang, L., Johansen, H.K., Ciofu, O., Hoiby, N., and Molin, S. (2012) Adaptation of Pseudomonas aeruginosa to the cystic fibrosis airway: an evolutionary perspective. Nat Rev Microbiol 10: 841-851.

Foxman, B. (2010) The epidemiology of urinary tract infection. Nat Rev Urol 7: 653-660.

Frohlich, F., Christiano, R., and Walther, T.C. (2013) Native SILAC: metabolic labeling of proteins in prototroph microorganisms based on lysine synthesis regulation. Mol Cell Proteomics 12: 1995-2005. Garbom, S., Olofsson, M., Bjornfot, A.C., Srivastava, M.K., Robinson, V.L., Oyston, P.C. et al. (2007) Phenotypic characterization of a virulence-associated protein, VagH, of Yersinia pseudotuberculosis reveals a tight link between VagH and the type III secretion system. Microbiology 153: 1464-1473. Geiger, T., Wisniewski, J.R., Cox, J., Zanivan, S., Kruger, M., Ishihama, Y., and Mann, M. (2011) Use of stable isotope labeling by amino acids in cell culture as a spike-in standard in quantitative proteomics. Nat Protoc 6: 147-157.

Giardina, G., Castiglione, N., Caruso, M., Cutruzzola, F., and Rinaldo, S. (2011) The Pseudomonas aeruginosa DNR transcription factor: light and shade of nitric oxide-sensing mechanisms. Biochem Soc Trans 39: 294-298.

Godeke, J., Binnenkade, L., and Thormann, K.M. (2012) Transcriptome analysis of early surfaceassociated growth of Shewanella oneidensis MR-1. PLoS One 7: e42160. 
Heurgue-Hamard, V., Champ, S., Engstrom, A., Ehrenberg, M., and Buckingham, R.H. (2002) The hemK gene in Escherichia coli encodes the N(5)-glutamine methyltransferase that modifies peptide release factors. EMBO J 21: 769-778.

Jander, G., Rahme, L.G., and Ausubel, F.M. (2000) Positive correlation between virulence of Pseudomonas aeruginosa mutants in mice and insects. J Bacteriol 182: 3843-3845. Jimenez, P.N., Koch, G., Thompson, J.A., Xavier, K.B., Cool, R.H., and Quax, W.J. (2012) The multiple signaling systems regulating virulence in Pseudomonas aeruginosa. Microbiol Mol Biol Rev 76: 46-65. John, M., Kudva, I.T., Griffin, R.W., Dodson, A.W., McManus, B., Krastins, B. et al. (2005) Use of in vivo-induced antigen technology for identification of Escherichia coli 0157:H7 proteins expressed during human infection. Infect Immun 73: 2665-2679.

Jung, J.S., Eberl, T., Sparbier, K., Lange, C., Kostrzewa, M., Schubert, S., and Wieser, A. (2014) Rapid detection of antibiotic resistance based on mass spectrometry and stable isotopes. Eur J Clin Microbiol Infect Dis 33: 949-955.

Kaplan, E.L., and Meier, P. (1958) Nonparametric Estimation from Incomplete Observations. Journal of the American Statistical Association 53: 457-481.

Kwon, T., Huse, H.K., Vogel, C., Whiteley, M., and Marcotte, E.M. (2014) Protein-to-mRNA ratios are conserved between Pseudomonas aeruginosa strains. J Proteome Res 13: 2370-2380.

Liberati, N.T., Urbach, J.M., Miyata, S., Lee, D.G., Drenkard, E., Wu, G. et al. (2006) An ordered, nonredundant library of Pseudomonas aeruginosa strain PA14 transposon insertion mutants. Proc Natl Acad Sci U S A 103: 2833-2838.

Little, R.H., Grenga, L., Saalbach, G., Howat, A.M., Pfeilmeier, S., Trampari, E., and Malone, J.G. (2016) Adaptive Remodeling of the Bacterial Proteome by Specific Ribosomal Modification Regulates Pseudomonas Infection and Niche Colonisation. PLoS Genet 12: e1005837. Lunter, G., and Goodson, M. (2011) Stampy: a statistical algorithm for sensitive and fast mapping of Illumina sequence reads. Genome Res 21: 936-939. 
Maier, T., Guell, M., and Serrano, L. (2009) Correlation of mRNA and protein in complex biological samples. FEBS Lett 583: 3966-3973.

Mora, L., Heurgue-Hamard, V., de Zamaroczy, M., Kervestin, S., and Buckingham, R.H. (2007)

Methylation of bacterial release factors RF1 and RF2 is required for normal translation termination in vivo. J Biol Chem 282: 35638-35645.

Nakahigashi, K., Kubo, N., Narita, S., Shimaoka, T., Goto, S., Oshima, T. et al. (2002) HemK, a class of protein methyl transferase with similarity to DNA methyl transferases, methylates polypeptide chain release factors, and hemK knockout induces defects in translational termination. Proc Natl Acad Sci U S A 99: 1473-1478.

Ong, S.E., Blagoev, B., Kratchmarova, I., Kristensen, D.B., Steen, H., Pandey, A., and Mann, M. (2002) Stable isotope labeling by amino acids in cell culture, SILAC, as a simple and accurate approach to expression proteomics. Mol Cell Proteomics 1: 376-386.

Park, Y., Yilmaz, O., Jung, I.Y., and Lamont, R.J. (2004) Identification of Porphyromonas gingivalis genes specifically expressed in human gingival epithelial cells by using differential display reverse transcription-PCR. Infect Immun 72: 3752-3758.

Ping, L., Zhang, H., Zhai, L., Dammer, E.B., Duong, D.M., Li, N. et al. (2013) Quantitative proteomics reveals significant changes in cell shape and an energy shift after IPTG induction via an optimized SILAC approach for Escherichia coli. J Proteome Res 12: 5978-5988.

Pustelny, C., Brouwer, S., Musken, M., Bielecka, A., Dotsch, A., Nimtz, M., and Haussler, S. (2013) The peptide chain release factor methyltransferase PrmC is essential for pathogenicity and environmental adaptation of Pseudomonas aeruginosa PA14. Environ Microbiol 15: 597-609.

Romeo, T., Vakulskas, C.A., and Babitzke, P. (2013) Post-transcriptional regulation on a global scale: form and function of Csr/Rsm systems. Environ Microbiol 15: 313-324.

Scarlett, D.J., McCaughan, K.K., Wilson, D.N., and Tate, W.P. (2003) Mapping functionally important motifs SPF and GGQ of the decoding release factor RF2 to the Escherichia coli ribosome by hydroxyl 
radical footprinting. Implications for macromolecular mimicry and structural changes in RF2.J Biol Chem 278: 15095-15104.

Schmidt, A., Kochanowski, K., Vedelaar, S., Ahrne, E., Volkmer, B., Callipo, L. et al. (2016) The quantitative and condition-dependent Escherichia coli proteome. Nat Biotechnol 34: 104-110. Schroder, A., Rohrbeck, A., Just, I., and Pich, A. (2015) Proteome Alterations of Hippocampal Cells Caused by Clostridium botulinum C3 Exoenzyme. J Proteome Res 14: 4721-4733.

Shi, J., Jin, Y., Bian, T., Li, K., Sun, Z., Cheng, Z. et al. (2015) SuhB is a novel ribosome associated protein that regulates expression of MexXY by modulating ribosome stalling in Pseudomonas aeruginosa. Mol Microbiol 98: 370-383.

Soufi, B., Kumar, C., Gnad, F., Mann, M., Mijakovic, I., and Macek, B. (2010) Stable isotope labeling by amino acids in cell culture (SILAC) applied to quantitative proteomics of Bacillus subtilis. J Proteome Res 9: 3638-3646.

UniProt, C. (2014) Activities at the Universal Protein Resource (UniProt). Nucleic Acids Res 42: D191198.

Ventre, I., Goodman, A.L., Vallet-Gely, I., Vasseur, P., Soscia, C., Molin, S. et al. (2006) Multiple sensors control reciprocal expression of Pseudomonas aeruginosa regulatory RNA and virulence genes. Proc Natl Acad Sci U S A 103: 171-176.

Wassarman, K.M. (2002) Small RNAs in bacteria: diverse regulators of gene expression in response to environmental changes. Cell 109: 141-144.

Winsor, G.L., Lam, D.K., Fleming, L., Lo, R., Whiteside, M.D., Yu, N.Y. et al. (2011) Pseudomonas Genome Database: improved comparative analysis and population genomics capability for Pseudomonas genomes. Nucleic Acids Res 39: D596-600.

Wu, L., Candille, S.I., Choi, Y., Xie, D., Jiang, L., Li-Pook-Than, J. et al. (2013) Variation and genetic control of protein abundance in humans. Nature 499: 79-82. 


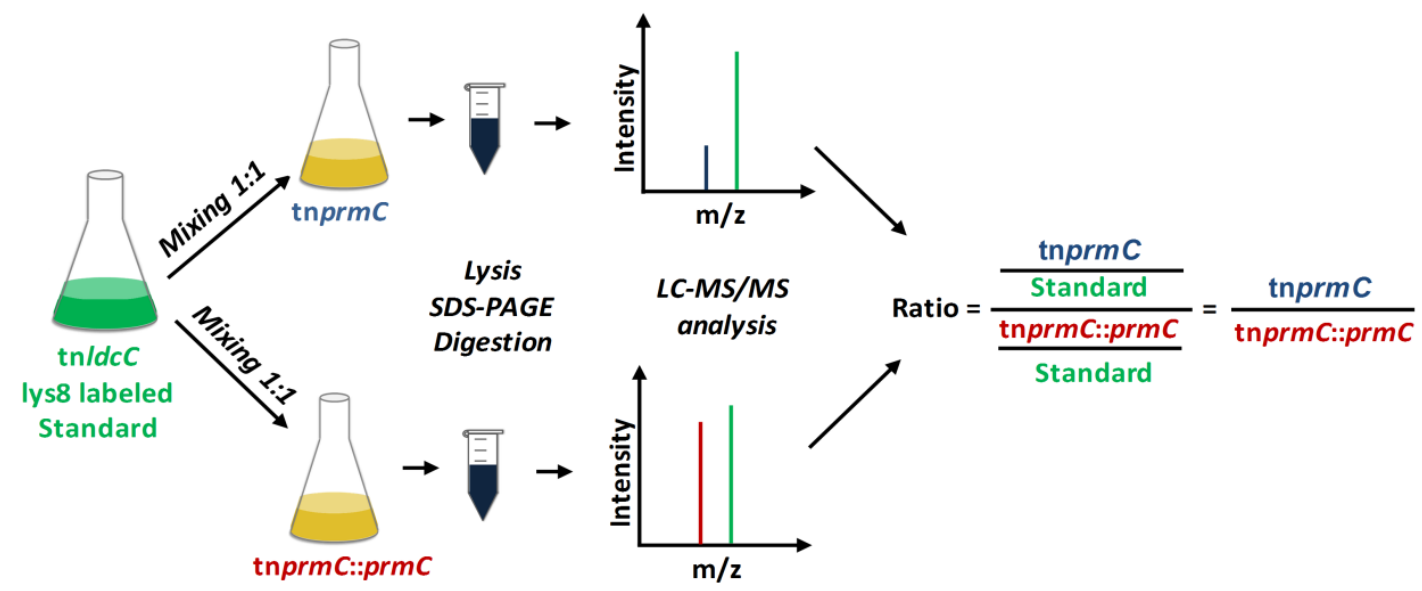

Figure 1. SILAC “spike in" experiment for global protein quantification in P. aeruginosa. For

quantitative proteome analysis the cultures of the PA14 tnprmC transposon mutant either the individual ratios to the "spike in" standard were combined. 


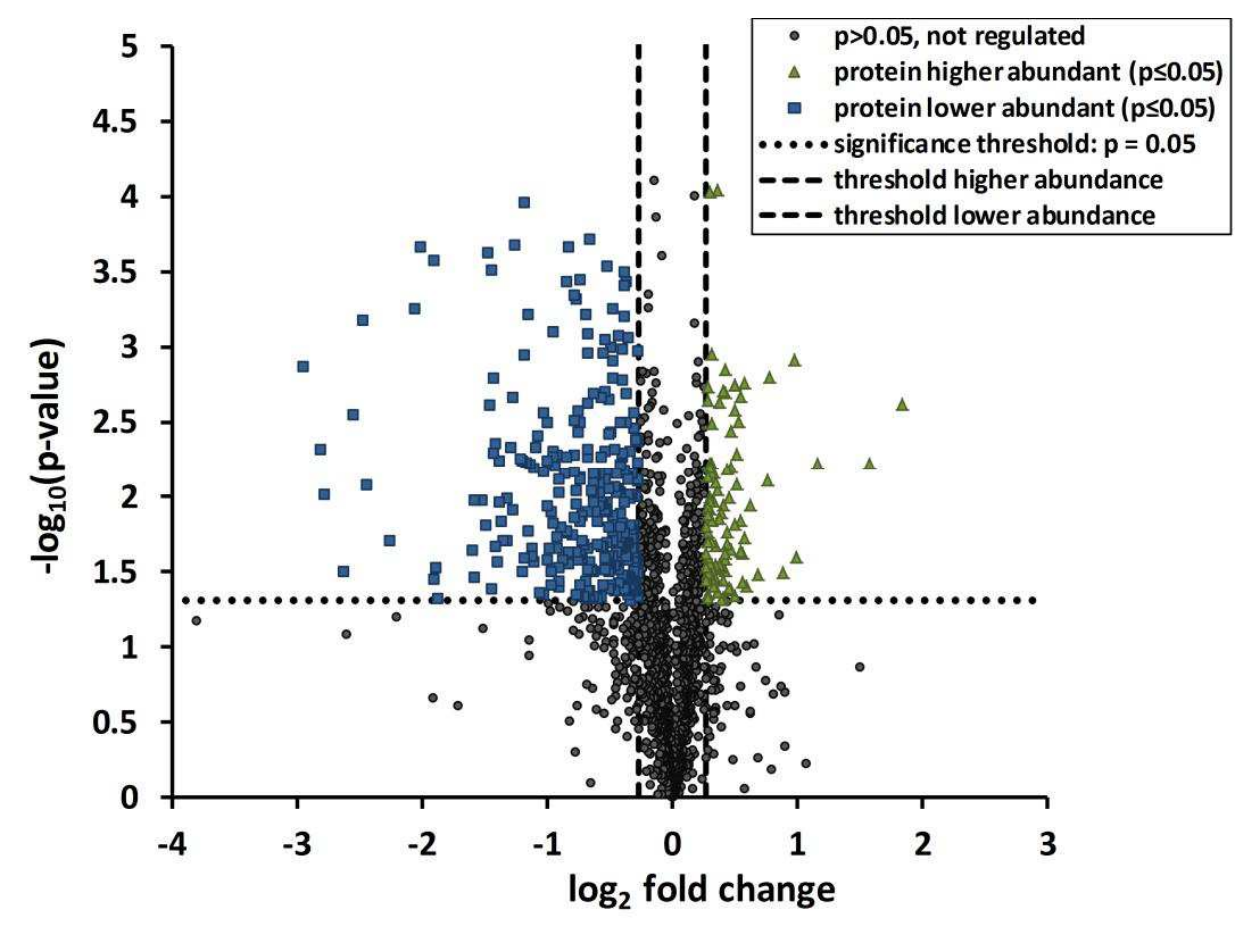

542 Figure 2. Volcano plot depicting PrmC-dependent changes of protein abundance in PA14. Squares

543 represent proteins which are significantly lower abundant in the PA14 tnprmC transposon mutant

544 complemented with pUCP20::EV (tnprmC) compared to PA14 tnprmC complemented with

545 pUCP20::prmC (tnprmC::prmC, Student's t-test $p \leq 0.05$ ) with a ratio (tnprmC/prmC::prmC) $\leq 0.83$.

546 Triangles represent proteins which are significantly higher abundant in the tnprmC mutant with a

547 ratio $\geq 1.2$. Circles represent proteins which have either no differential abundance (ratio $<1.2$; ratio

$548>0.83)$ or exhibit no significant change $(p>0.05)$ in abundance. $n=3$ for this experiment. 


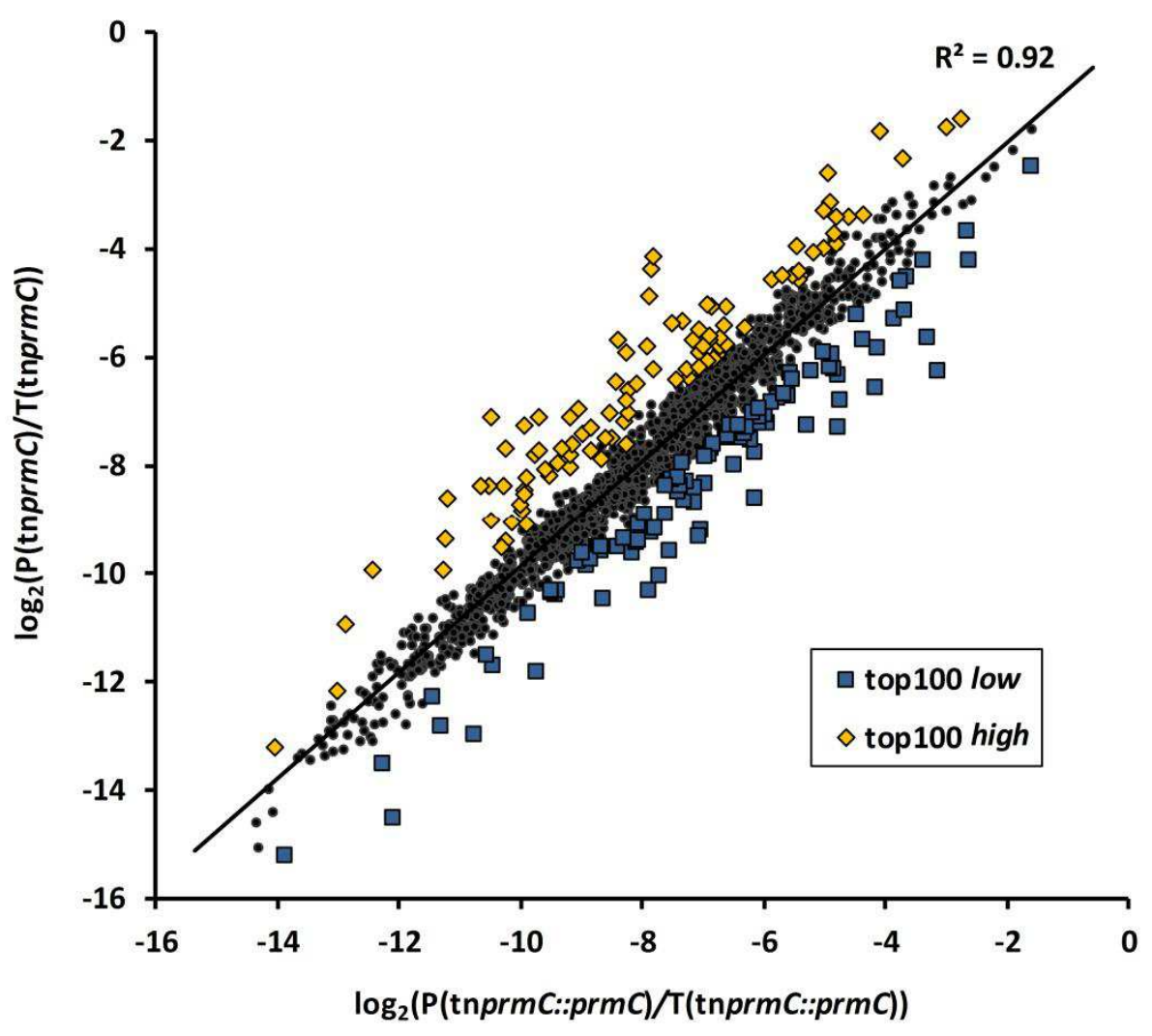

550

551 Figure 3. Correlation of PrmC-dependent protein to mRNA ratios. The protein $(\mathrm{P})$ to mRNA $(\mathrm{T})$ ratios 552 of the PA14 tnprmC transposon mutant either complemented with pUCP20::EV (tnprmC) or 553 pUCP20::prmC (tnprmC::prmC) were calculated and plotted against each other. Most of the protein 554 to mRNA ratios did not significantly differ between the two strains, indicating that the ratios were 555 independent of the presence of PrmC. PrmC-dependent protein to mRNA ratios were calculated by 556 dividing protein ratios (tnprmC/tnprmC::prmC) by mRNA ratios. Squares represent the 100 proteins 557 exhibiting the lowest values for the ratio between protein to mRNA ratios (top100 low, S3 Table). 558 Diamonds represent 100 proteins exhibiting the highest values for the ratio between protein to 559 mRNA ratios (top100 high, S4 Table). $\mathrm{R}^{2}$ indicates squared Pearson's correlation coefficient. 


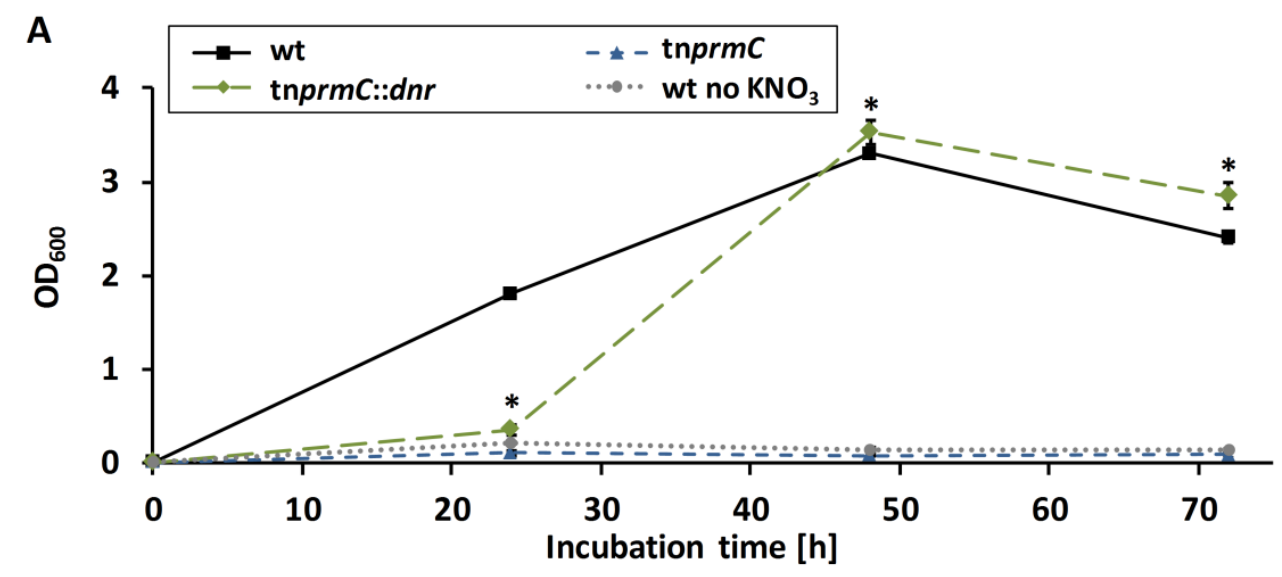

B

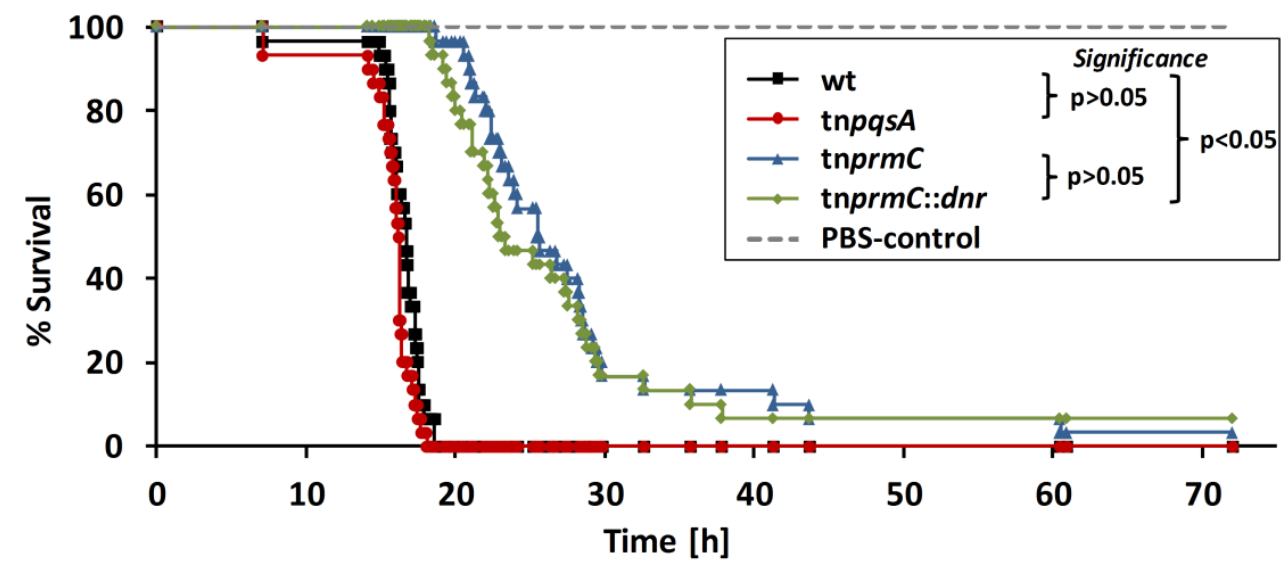

562 Figure 4. Complementation of the tnprmC mutant by DNR. (A) For monitoring PrmC and DNR dependent anaerobic growth behavior in Pyg medium, $\mathrm{OD}_{600}$ was measured. Anaerobic cultures were inoculated by PA14 wt or tnprmC strains complemented either with pUCP20::EV or pUCP20::dnr. For negative control the wt strain was cultivated without $\mathrm{KNO}_{3} ; \mathrm{n}=3$ for the experiment. Data points are presented as mean \pm SD. The * indicates significantly altered values between tnprmC::EV and tnprmC::dnr with a p-value $\leq 0.05$ as determined by Student's t-test. (B) To test the PrmC and DNR dependent pathogenicity of PA14 the Galleria mellonella virulence assay (Jander et al., 2000) was applied with minor changes. Larvae were infected with 50 cells of PA14 wt, tnpqsA or tnprmC complemented either with pUCP20::EV or pUCP20::dnr or PBS as negative control. Survival rates of

57130 larvae per strain (10 per replicate, $n=3$ ) were determined by video monitoring. Mortality time

572 point was asserted by melanization of the cuticle and lack of movement for at least 20 min.

573 Significance was determined by Kaplan Meier estimation (Kaplan and Meier, 1958). 


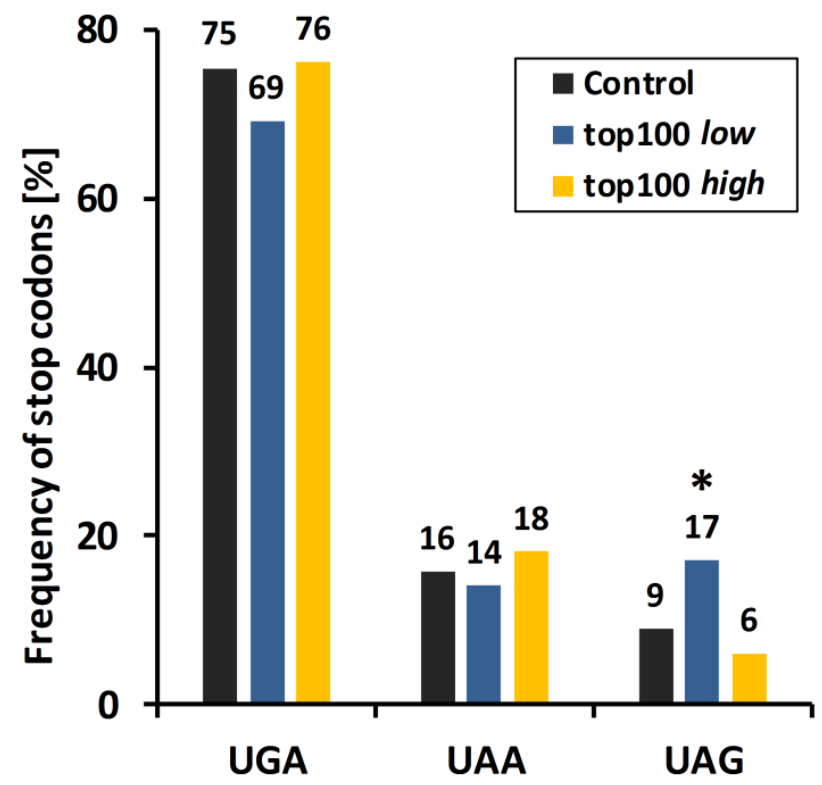

574

575 Figure 5. Stop codon usage is responsible for PrmC-dependent changes in the translation efficiency.

576 An enrichment analysis for the three stop codons UGA, UAA and UAG was performed in the top100

577 low and the top100 high groups (as defined in Fig. 3) in comparison to all genes quantified on the

578 protein level as the control. The * indicates significantly altered values with a $p$-value $\leq 0.05$ as

579 determined by hypergeometric distribution. 